\title{
Etude et dimensionnement des digues à talus Prise en compte du caractère aléatoire de la houle
}

\author{
Iskander Tlili $^{1^{*}}$, Mohamed Ayari ${ }^{2}$ \\ ${ }^{1}$ Laboratoire d'Etude des Systèmes Thermiques et Energétiques. Ecole Nationale \\ d'Ingénieurs de Monastir Rue Ibn El Jazzar. 5019 Monastir. Tunisie. \\ 2 Ministère de l'équipement, de l'habitat et de l'aménagement du territoire. \\ Direction générale de service aérienne et maritime \\ * Corresponding author \\ E-mail : Iskander.Tlili@enim.rnu.tn \\ Tel: 0021698619704 \\ Fax: 0021673500514
}

\section{Résumé}

Les études et les dimensionnements des digues à talus sont le plus souvent réalisées en laboratoire, dans des canaux à houle bidimensionnels équipés à une extrémité d'un dispositif générateur de vagues et à l'autre d'un dispositif amortisseur de type plage artificielle, ou autre. Ce confinement en espace engendre des phénomènes parasites, non présents en milieu ouvert, qui vont perturber l'écoulement. Aussi les modèles mathématiques ne traduit pas réellement la réalité comme elles sont basées sur des formules empiriques qui ne prennent pas en compte le caractère aléatoire des houles. Dans cet article Nous allons successivement passer en revue ces différents points en cherchant à préciser l'influence des paramètres les moins bien connus à travers les études effectuées ces dernières années et de comparer l'effet de la houle aléatoire à celle régulière.

\begin{abstract}
The studies and dimensioning of the dams with slope are generally carried out in laboratory, in two-dimensional channels with swell equipped at an end with a generating device with waves and with the other with a cushioning device of the type artificial beach, or other. This containment in space generates parasitic phenomena, not present in opened medium, which will disturb the flow. Also the mathematical model does not correspond to really reality as they are based on empirical formulas which do not take into account the randomness of the swells. In this article We successively will review these various points while seeking to specify the influence of the parameters well-known through the studies carried out these last years and to compare the effect of the random swell with that regular
\end{abstract}

\section{Mots clés}

Digue à talus / dimensionnement / houle de projet 


\section{$\underline{\text { 1. Introduction }}$}

L'homme a de tout temps utilisé les mers comme voie de communication et a simultanément été obligé de créer des abris pour assurer la liaison terre-mer. Il a également dû défendre son territoire contre les assauts de la mer, de construire des ouvrages portuaires et d'aménager son littoral. Or cet espace d'interface entre la mer et la terre est fragile, il est le siège d'un équilibre souvent précaire entre différents facteurs naturel est humains. Ainsi, son importance dans notre environnement et sa sensibilité aux différentes interventions dont il peut être le théâtre font qu'il est nécessaire qu'il soit protégé ou aménagé avec respect. Afin de protéger les côtes des effets destructeurs de la houle, l'homme construit des dispositifs capables de dissiper l'énergie des vagues. Ces dispositifs sont en générales les digues à talus qui présentent des avantages importantes, relativement simple à construire, présente la particularité de ne subir que des destructions progressives comme ils sont relativement peu réfléchissant $(30 \%$ à $50 \%$ environ selon la cambrure). Plusieurs études de dimensionnement des digues à talus ont été réalisés [3-7] on distingue les modèles réduits physiques, les modèles mathématiques et les modèles hybrides. Par rapport au modèle physique, le modèle mathématique permet de couvrir des zones géographiques très grandes, son encombrement est nul, son exploitation est plus rapide et il peut être conservé, ce qui permet sa réutilisation ultérieure. Mais les modèles mathématiques ne traduit pas précisément la réalité, les laboratoires utilisent la formule d'Hudson qui a cependant été établie à partir d'essais réalisés sur des digues en enrochements naturels en houle régulière non déferlante, par conséquent dans des conditions assez éloignées des conditions réelles auxquelles pourrait être soumis l'ouvrage. Des recherches récentes établissent des nouvelles formules incluent plusieurs paramètres tel que la période des vagues, durée de la tempête, perméabilité de la structure et le niveau de dommage.

\section{$\underline{\text { 2. Etat de la connaissance des facteurs naturels mise en jeu }}$}

La mise au point des digues à talus nécessite une connaissance approfondie des facteurs naturels et notamment des divers mouvements de la mer.

\subsection{Marée}

La marée est connue de façon assez précise déjà depuis longtemps et les théories élaborées pour analyser ce phénomène ont permis d'établir des annuaires donnants, avec une approximation souvent suffisante, les prévisions de son amplitude pour chaque jour en de nombreux point du globe.

\subsection{Courants}

Les courant marins généraux et les courants de marée sont plus constants, quoique ces derniers varient d'une marée à l'autre, mais leur mesure est plus aisée et leur connaissance en une zone donnée relativement facile à déterminer. 


\subsection{Houle}

La houle est par contre un phénomène beaucoup plus aléatoire aussi bien dans le temps qu'en direction et intensité. Elle ne peut être connue que statistiquement dans une zone donnée. Sa déformation au voisinage des côtes nécessite qu'elle soit mesurée à une certaine distance de rivage.

Finalement le facteur le plus important dans la plupart des études maritimes est la houle qu'est le plus difficile à connaître d'où la nécessité de déterminer la houle de projet.

\section{Détermination de la houle de projet}

\subsection{Cas des eaux peu profondes :}

Pour déterminer la houle de dimensionnement d'un ouvrage implanté à des petites profondeurs, on peut exploiter un résultat de déferlement des houles qui considère que la hauteur d'une vague ne peut pas évoluer indépendamment de la hauteur du tirant d'eau dans lequel elle se propage.

\subsubsection{Houles régulières :}

Les premières approches considèrent que le déferlement d'une houle se propageant sur un fond de profondeur $\mathbf{d}$ de pente $\mathrm{m}$ commence dès que sa hauteur $\mathbf{H}$ atteint une valeur tel que :

$$
\frac{H}{d}=\frac{1,56}{\left[1+e^{-19,5 m}\left[1+4,41\left(1-e^{-19 m}\right) \frac{d}{T^{2}}\right]\right.}
$$

Dont on peut déduire la hauteur $\mathrm{H}$ que la houle ne peut pas dépasser.

En particulier si la pente $\mathrm{m}$ est inférieure à $2 \%, \mathrm{H} \approx 0,8 \mathrm{~d}$ ( MUK 1949) [2].

Toute fois il s'est avéré suite à des études que la hauteur de déferlement des vagues dépend de cambrure

Lorsque l'ouvrage à étudier est implanté au faible profondeur, le gonflement des vagues n'obéit plus au loi de Schoaling déduite de la théorie linéaire de la houle mais est aussi fonction de la cambrure de la houle et de la pente moyenne des fonds $\mathrm{m}$ devant l'ouvrage.

\subsubsection{Houles irrégulières:}

Les abaques élaborés par GODA [2] donnant les hauteurs maximales et significatives des houles réelles déferlantes en fonction de la pente et la hauteur

\section{Comparaison de l'évolution des dommages en houle aléatoire et en houle régulière}

\subsection{Résultats obtenus pour des digues en enrochements naturels}


Par souci de clarté, on peut distinguer 3 étapes parmi les résultats obtenus :

- la première a consisté naturellement à comparer les résultats de LNH et les résultats d'Hudson pour quelques essais réalisés en houle régulière.

- La seconde a permis de comparer les résultats d'Hudson en houle régulière et les résultats obtenus en houle aléatoire pour une durée de tempête fixée.

- La troisième étape a permis de préciser l'influence de la durée de tempête sur la stabilité des ouvrages.

\subsubsection{Résultats d'Hudson :}

Les conditions d'essais réalisés par Hudson sont reportées et comparées dans le tableau I aux conditions d'essais réalisés en houle régulière au L.N.H. Les valeurs indiquées sont à l'échelle 1 :

\begin{tabular}{|l|l|l|}
\hline & $\begin{array}{l}\text { Waterways Experiment } \\
\text { Station (Hudson ) }\end{array}$ & L.N.H. \\
\hline Hauteur de houle & $8 \mathrm{~cm} \rightarrow 20 \mathrm{~cm}$ & $6 \mathrm{~cm} \rightarrow 13 \mathrm{~cm}$ \\
\hline $\begin{array}{l}\text { Profondeur d'eau au } \\
\text { Pied de l'ouvrage }\end{array}$ & $40 \mathrm{~cm} \rightarrow 60 \mathrm{~cm}$ & $8 \mathrm{~cm} \rightarrow 30 \mathrm{~cm}$ \\
\hline Période de la houle & $0.9 \mathrm{~s} \rightarrow 2.5 \mathrm{~s}$ & $0.9 \mathrm{~s} \rightarrow 1.9 \mathrm{~s}$ \\
\hline Poids des blocs & $40 \mathrm{~g} \rightarrow 140 \mathrm{~g}$ & $62 \mathrm{~g} \rightarrow 94 \mathrm{~g}$ \\
\hline Pente de l'ouvrage & $1.25 \rightarrow 5$ & $1.5 \rightarrow 2$ \\
\hline $\begin{array}{l}\text { Pente des fonds } \\
\text { Devant la digue }\end{array}$ & 0 & $0.05 \rightarrow 0.1$ \\
\hline
\end{tabular}

Tableau 1 : Résultats comparatives Hudson L.N.H.

Cette comparaison met en évidence deux caractéristiques importantes des essais d'Hudson :

- $\quad$ la profondeur d'eau est grande devant la hauteur de houle.

- $\quad$ la pente des fonds devant l'ouvrage est nulle.

Une analyse sommaire des conditions d'équilibre d'un bloc sous l'action de son poids, des forces d'inertie et des forces de traînée (ces dernières étant calculées en supposant que la vitesse du jet de la vague déferlante est de la forme $\mathrm{k} \sqrt{\mathrm{gH}}$ ) conduit à la relation (Hudson) :

$$
\frac{\gamma_{\mathrm{r}}^{1 / 3_{\mathrm{H}}}}{(S r-1) \mathrm{W}_{\mathrm{r}}^{1 / 3}}=\mathrm{f}\left(\alpha, \frac{\mathrm{H}}{\mathrm{L}}, \frac{\mathrm{Dp}}{\mathrm{L}}, \mathrm{D}, \text { forme des blocs }\right)
$$

$\gamma_{\mathrm{r}}$ est la masse volumique du matériau utilisé.

$\mathrm{W}_{\mathrm{r}}$ est le poids unitaire des blocs.

Sr est la densité du matériau par rapport à l'eau.

$\mathrm{H}$ est la hauteur de vague.

$\alpha$ est l'angle du talus de la digue.

Dp est la profondeur d'eau au pied de la digue.

L est la longueur d'onde de la houle.

D sont des dommages exprimant le rapport du nombre de blocs déplacés au nombre de blocs total de la carapace. 
Le terme de gauche de cette relation est un nombre adimensionnel appelé nombre de stabilité et noté Ns.

La liste des variables de la fonction $\mathrm{f}$ du terme de droite de la relation précédente n'est évidemment pas exhaustive. Elle ne tient compte que des paramètres non fixés dans la réalisation des essais présentés ici. Cette fonction dépend également du nombre de couches de la carapace (égale à 2 ici), du plan de pose des blocs (dans le cas de blocs artificiels de forme complexe), de l'indice de vide, de la pente de fonds devant l'ouvrage etc.

Le principal intérêt des résultats d'Hudson est d'avoir permis de préciser la fonction $\mathrm{f}$ et d'avoir établi une relation liant le nombre Ns aux dommages admis $\mathrm{D}$, la pente du talus $\alpha$ et au type de blocs utilisé (mais seulement pour ce dernier paramètre dans le cas de tétrapodes et d'enrochements naturels).

La relation obtenue expérimentalement est de la forme :

$$
\mathrm{Ns}=\sqrt[3]{\mathrm{k}_{\mathrm{D}} \cot g \alpha}
$$

$\mathrm{k}_{\mathrm{D}}$ étant une fonction des dommages admis et de type de blocs utilisé.

Pour un type d'ouvrage donné ( pente du talus, type de blocs choisis) et pour des dommages admissibles fixés, le nombre Ns est une constante, ce qui fixe également, pour une densité de matériau donnée le rapport $\mathrm{H} / \mathrm{W}^{1 / 3}$. Par conséquent à une hauteur de houle donnée on pourra faire correspondre un poids de blocs $\mathrm{W}$ d'autant plus faible que la valeur de l'expression $\mathrm{Ns}=\sqrt[3]{\mathrm{k}_{\mathrm{D}} \cot g \alpha}$ est élevée.

Les variations de $\mathrm{k}_{\mathrm{D}}$ pour chaque tranche de dommages sont données dans le tableau ci-dessous toujours pour des enrochements naturels.

\begin{tabular}{|c|c|}
\hline Dommages \% & $\mathrm{k}_{\mathrm{D}}$ \\
\hline $0-1$ & 3.2 \\
$1-5$ & 5.1 \\
$5-10$ & 7.2 \\
$10-20$ & 9.5 \\
$15-40$ & 12.8 \\
\hline
\end{tabular}

Tableau 2: Valeurs données par Hudson

Ces résultats importants montrent que dans les conditions d'essais décrites cidessus (pas de déferlement avant ou sur l'ouvrage notamment) l'influence de la période de la houle est négligeable et que le nombre de stabilité Ns est lié essentiellement au type de blocs choisi, à la pente de l'ouvrage et aux dommages admissibles.

Rappelons que ces essais ont été effectués en houle régulière et en absence de déferlement. Une méthode, résultant d'essais réalisés au L.N.H. et permettant de prendre en compte le caractère aléatoire de la houle sera développée dans les paragraphes suivants.

\subsubsection{Résultats obtenus en houle régulière au L.N.H :}

Les résultats obtenus en houle régulière au L.N.H. et présentés sur la figure 5 corroborent les résultats d'Hudson pour des digues à talus de pente $3 / 2$ et 2 . Seuls les résultats d'essais effectués dans des conditions proches de celles d'Hudson ont 
été reportés dans cette figure (pente de fonds faible, profondeur d'eau importante au pied de la digue $(15$ à $30 \mathrm{~cm})$.
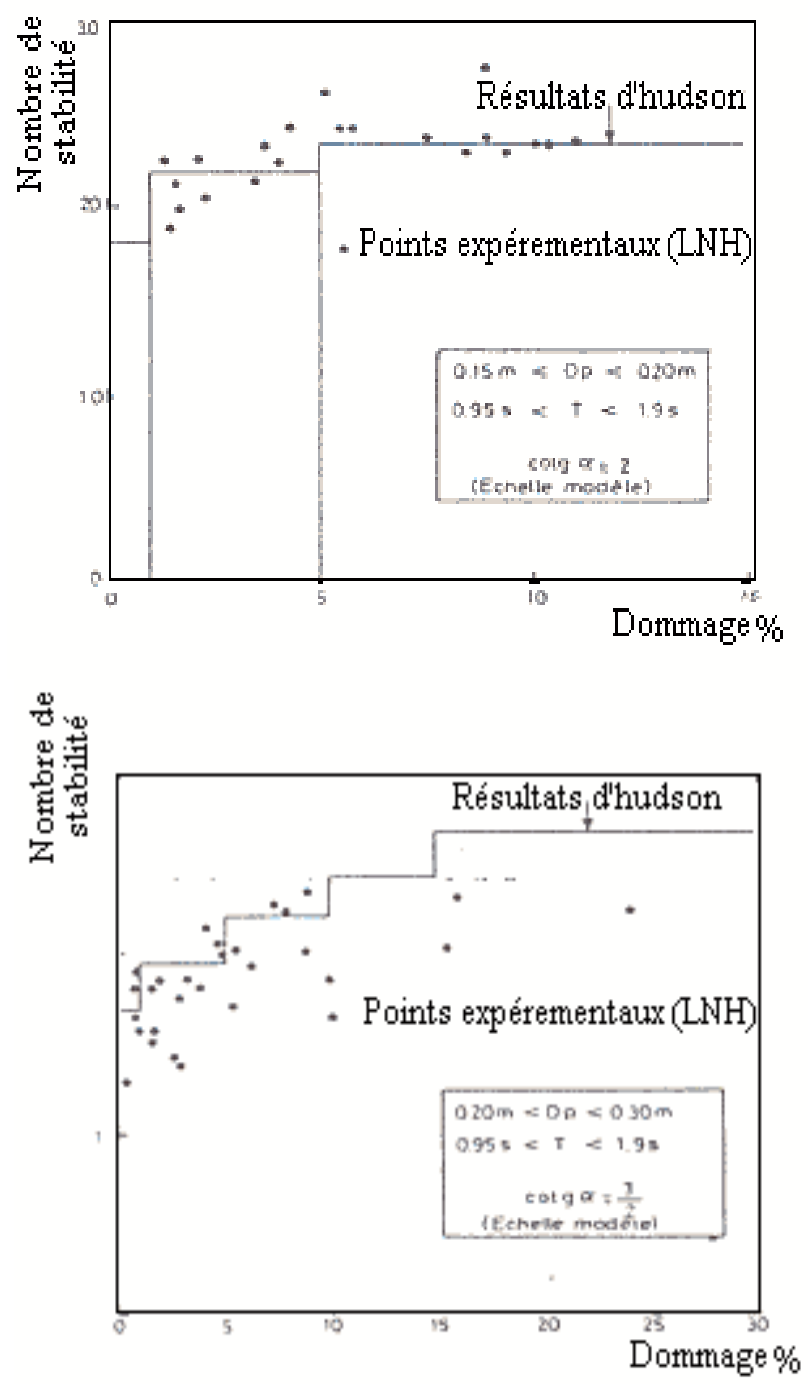

Figure 1 : Nombre de stabilité Hudson- L.N.H.

Malgré une dispersion importante des points, la conclusion énoncée ci-dessus semble à peu près indépendante de la période de la houle dans la gamme de périodes explorées.

\subsubsection{Comparaison des résultats obtenus en houle régulière et aléatoire pour une durée de tempête de 6 heures}

Les dommages mesurés en houle aléatoire sont comparés aux résultats d'Hudson. Pour cela le nombre de stabilité Ns a été calculé en utilisant différentes hauteurs de houle caractéristiques du spectre réparties dans la gamme $\overline{\mathrm{H}_{\mathrm{Z}}, \frac{1}{3}}, \mathrm{H}_{\max }$ 
$\left.\overline{\left(\mathrm{H}_{\mathrm{Z}}, \frac{1}{10}\right.} ; \overline{\mathrm{H}_{\mathrm{Z}}, \frac{1}{20}} ; \overline{\mathrm{H}_{\mathrm{Z}}, \frac{1}{100}}\right)$ et les valeurs obtenues sont comparées aux résultats d'Hudson à dommage égal.

Pour une durée de tempête de 6 heures un ajustement statistique de l'ensemble des résultats montre que le meilleur accord est obtenu en utilisant la hauteur $\overline{\mathrm{H}_{\mathrm{Z}}, \frac{1}{20}}$.

Ce résultat signifie que l'action pendant 6 heures d'une houle aléatoire de répartition spectrale de type Jonswap est équivalente en ce qui concerne les dommages à une houle régulière de $\overline{\mathrm{H}_{\mathrm{Z}}, \frac{1}{20}}$ agissant jusqu'à stabilisation de l'ouvrage.

Ce résultat est cependant à utiliser avec précaution et doit être modulé, notamment en fonction de la durée des tempêtes qui semble avoir une influence importante sur la stabilité des ouvrages. Dans le but de préciser ce point d'autres séries d'essais ont été effectuées en houle aléatoire pour des durées d'action de la houle comprises entre une et 20 heures.

\subsubsection{Application à la détermination de la hauteur de houle de dimensionnement $\mathbf{H}_{\mathrm{D}}$ :}

Les résultats présentés ci-dessus conduisent pour une durée de tempête de 6 heures à la relation :

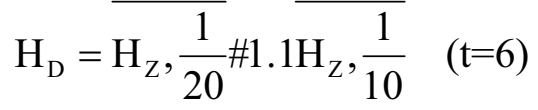

Exprimant l'équivalence pour les dommages entre une houle régulière de hauteur $\mathrm{H}_{\mathrm{D}}$

Agissant jusqu'à stabilisation de la destruction de l'ouvrage et une houle aléatoire de hauteur caractéristique $\overline{\mathrm{H}_{Z}, \frac{1}{20}}$ égale à $\mathrm{H}_{\mathrm{D}}$ agissant pendant 6 heures.

Le problème est alors de déterminer la hauteur de houle de dimensionnement $\mathrm{H}_{\mathrm{D}}$ pour une tempête de hauteur caractéristique $\overline{\mathrm{H}_{\mathrm{Z}}, \frac{1}{10}}$ et de durée $\mathrm{t}_{\mathrm{o}}$. les dommages à l'instant to seront :

$$
\mathrm{D}_{1}\left(t_{0}\right)=k \overline{\mathrm{H}}_{\mathrm{Z}}, \frac{1}{10} t_{0}^{(1) 3.9}
$$

Nous associerons à cette loi d'évaluation une autre loi d'évaluation des dommages :

$$
\mathrm{D}_{2}(t)=k \overline{\mathrm{H}}_{\mathrm{Z}}, \frac{1}{10}^{(2) 3.9} t^{0.37}
$$

Soit :

$$
H_{D}=f(t) \overline{H_{Z}, \frac{1}{10}} \quad \text { avec } f(t)=0.9 t^{0.1}
$$


Les variations de la hauteur de houle de dimensionnement avec la durée de la tempête ( pour une durée supérieure à 3 heures) sont relativement peu importantes mais ces résultats conduisent cependant à une augmentation importante du poids des blocs du talus, ce dernier étant proportionnel au cube de la hauteur de houle de dimensionnement (Voir figure suivante)

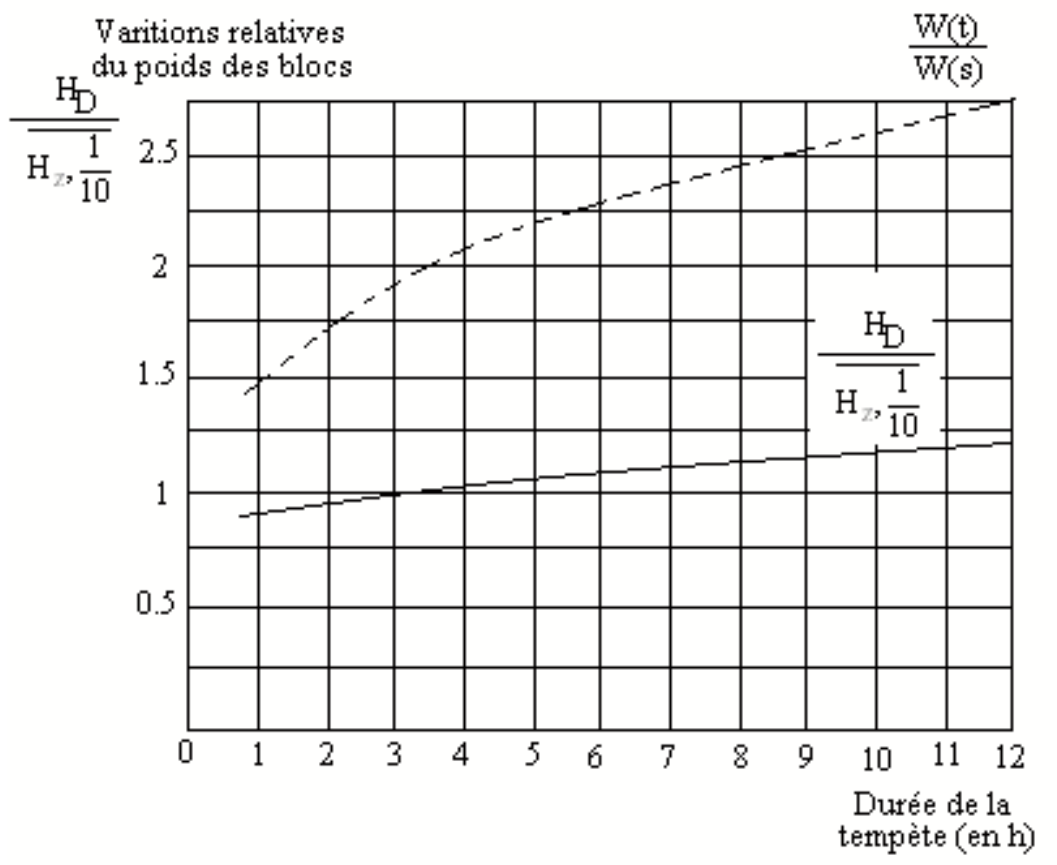

Figure 2 : Poids des blocs en fonction de la durée de la tempête

\section{Conclusion}

De nos jours la protection des ports vis-à-vis de la houle venant du large se fait par des ouvrages de plus en plus complexes capables de s'avérer à la fois peu réfléchissants et très atténuateurs. Pour respecter des cahiers des charges très exigeants, des mesures fiables de la réflexion et de la transmission sont donc nécessaires lors de l'étude de ces ouvrages

Nous montrons qu'à hauteur significative égale, la houle aléatoire est plus agressive que la houle régulière. Il faut noter que les essais ont été effectués avec un seul type de spectre (JONSWAP). Ce spectre est relativement étroit (largeur $\mathrm{e}=0.648$ ).

D'autres auteurs (CARSTEN-TORUM-TRAETTEBERG) [7], avec le même type de spectre, ont également montré que la hauteur caractéristique de houle à prendre en compte pour le dimensionnement des digues était supérieure à $\mathrm{H}_{1 / 3}$. Mais avec des types de spectres plus larges (Neuman) cette hauteur se rapprocherait de $\mathrm{H}_{1 / 3}$, comme en houle régulière. Cela paraît paradoxal. Nous pensons que l'explication peut être cherchée d'une part dans l'influence de la période, ou plus exactement dans l'existence d'un couple critique hauteur période, d'autre part dans l'existence de groupement de vagues, on sait en effet que plus un spectre est étroit plus les 
groupements de vagues sont important. Or ceux-ci sont réputés plus dommageables pour la structure (Mansard, Ploeg, Burcharth).

On a en particulier souvent constaté dans ces essais que l'attaque la plus forte se produisait lorsque la descente d'une vague coïncidait avec l'arrivée au pied de la digue du creux de la vague suivante. En effet, c'est dans cette situation que l'on doit avoir les vitesses maximales de la masse d'eau et sans doute les souspressions maximales sur les blocs. Cette situation dépend de la hauteur de la vague et de sa période, mais aussi de la pente du talus ainsi que de la rugosité et de la porosité de la carapace.

\section{Bibliographie}

1 Hudson R.Y. (1959). Laboratory investigation on rubble mound breakwaters. Journal of the waterways and harbors divisions. Proc. ASCE.

2 Chanson, H. (1994). Hydraulic design of stepped cascades, channels, weirs and pillways. Book, Pergamon Edition.

3 Chanson, H. (1993). Réduction des pertes de frottement pour des écoulements à surface libre avec entraînement d'air. Augmentation de la débitance d'un coursier d'évacuateur de crues. La Houille Blanche, 48 (5), pp.303-313.

4 Masbernat. L . (2003). Dynamique des ondes de surface. Cours à l'ENIT.

5 Sfar. A. (2003). Hydraulique maritime. Cours à l'ENIT.

7. Dubois, J.(1998), Comportement hydraulique et modélisation des écoulements de surface. Thèse $\mathrm{N}^{\circ} 1890$, EPF-Lausanne. 